\title{
Locally projective graphs and their densely embedded subgraphs
}

\section{Alexander A. Ivanov ${ }^{1,2,3}$}

Received: 28 January 2020 / Accepted: 25 November 2020 / Published online: 22 December 2020 (c) The Author(s) 2020

\begin{abstract}
The article contributes to the classification project of locally projective graphs and their locally projective groups of automorphisms outlined in Chapter 10 of Ivanov (The Mathieu Groups, Cambridge University Press, Cambridge, 2018). We prove that a simply connected locally projective graph $\Gamma$ of type $(n, 3)$ for $n \geq 3$ contains a densely embedded subtree provided (a) it contains a (simply connected) geometric subgraph at level 2 whose stabiliser acts on this subgraph as the universal completion of the Goldschmidt amalgam $G_{3}^{1} \cong\left\{S_{4} \times 2, S_{4} \times 2\right\}$ having $S_{6}$ as another completion, (b) for a vertex $x$ of $\Gamma$ the group $G_{\frac{1}{2}}(x)$ which stabilizes every line passing through $x$ induces on the neighbourhood $\Gamma(x)$ of $x$ the (dual) natural module $2^{n}$ of $G(x) / G_{\frac{1}{2}}(x) \cong L_{n}(2)$, (c) $G(x)$ splits over $G_{\frac{1}{2}}(x)$, (d) the vertex-wise stabilizer $G_{1}(x)$ of the neighbourhood of $x$ is a non-trivial group, and (e) $n \neq 4$.
\end{abstract}

\section{Introduction}

We start with our principal definitions.

Definition 1 Let $\Gamma$ be a connected graph and let $G$ be a group of automorphisms of $\Gamma$. Then $\Gamma$ is said to be locally projective of type $(n, \alpha)$, where $n \geq 2, \alpha \in\{2,3\}$ with respect to the action of $G$, whenever the following conditions hold:

(i) $G$ acts vertex- and edge-transitively on $\Gamma$;

(ii) there is a family $\mathcal{L}$ of complete subgraphs in $\Gamma$ (called lines) having $\alpha$ vertices each, such that $(a) \mathcal{L}$ is preserved by $G$, and $(b)$ every edge of $\Gamma$ is contained in a unique line from $\mathcal{L}$;

\footnotetext{
Alexander A. Ivanov a.ivanov@ic.ac.uk

1 Imperial College London, London, UK

2 Three Gorges Mathematical Research Center, Yichang, Hubei, China

3 Institute for System Analysis ERC, CSC RAS, Moscow, Russia
} 
(iii) every vertex $x$ of $\Gamma$ is contained in exactly $\left(2^{n}-1\right)$ lines and the stabilizer $G(x)$ of $x$ in $G$ induces on this $\left(2^{n}-1\right)$-set of lines the natural doubly transitive action of the group $L_{n}(2)$ as on the set of points of the corresponding projective $G F(2)$-geometry $\pi_{x}$;

(iv) the stabilizer in $G$ of a line acts doubly transitively on vertex-set of the line;

(v) if $\alpha=2$ then $G$ is not transitive on 3-paths in $\Gamma$ and whenever $\{x, y\}$ is an edge, an element swapping $x$ and $y$ induces a collineation (rather than correlation) between the residue of $y$ in $\pi_{x}$ and the residue of $x$ in $\pi_{y}$.

If $\alpha=2$, then $\mathcal{L}$ is the edge-set of $\Gamma$. If $\alpha=3$, then $\mathcal{L}$ is a family of triangles in $\Gamma$; the stabilizer $G(l)$ of a line-triangle $l$ induces on its vertices the symmetric group $S_{3} \cong L_{2}$ (2). Since by (ii)(b) any two lines intersect in at most one vertex, the valency of $\Gamma$ is $2 \cdot\left(2^{n}-1\right)$. The $G F(2)$-vector space whose non-zero vectors are indexed by the lines passing through $x$ will be called the natural module of the group $L_{n}(2)$ induced by $G(x)$ on the set of these lines.

Definition 2 Let $\Gamma$ be a graph which is locally projective of type $(n, \alpha)$ with respect to a group $G$. Let $x$ be a vertex of $\Gamma$ and let $l$ be a line containing $x$. Then the amalgam

$$
\mathcal{A}=\{G(x), G(l)\}
$$

is said to be a locally projective amalgam of type $(n, \alpha)$.

The vertex stabilizers in locally projective graphs of type $(2,2)$ were classified by Sims (1967). This result motivated him to state the famous Sims conjecture. This was extended to the classification of locally projective amalgams of type $(2,2)$ by Djoković and Miller (1980). The classification of the amalgams of type $(2,3)$ is the main result of Goldschmidt's paper (Goldschmidt 1980), which is the most influential publication of the second quarter of the 20-th century in finite group theory for it has put the foundation of the amalgam method as we know it now.

The locally projective amalgams of type $(n, 2)$ for all $n \geq 3$ were classified by Shpectorov and the present author (Ivanov and Shpectorov 2004) making use of a fundamental result by Trofimov (2003). The classification is given in the following theorem, where $G_{i}(x)$ denotes the vertex-wise stabilizer in $G$ of the ball of radius $i$ in $\Gamma$ centered at $x$ and $V_{i}=G_{i}(x) / G_{i+1}(x)$.

Theorem 3 Ivanov and Shpectorov (2004) Let $G$ be a group acting locally projectively on a graph $\Gamma$ of type $(n, 2)$ for some $n \geq 3$, and let $\mathcal{A}=\{G(x), G(l)\}$ be the corresponding locally projective amalgam. Then one of the following three possibilities holds:

(i) $\mathcal{A}$ is isomorphic to the locally projective amalgam associated with the natural action of the affine group $A G L_{n}(2)$ on the vector-set of the corresponding $n$ dimensional $G F(2)$-space;

(ii) $\mathcal{A}$ is isomorphic to the locally projective amalgam associated with the natural action of the orthogonal group $\mathrm{O}_{2 n}^{+}(2)$ on the corresponding dual polar space graph;

(iii) $\mathcal{A}$ is one of the twelve exceptional amalgams in Table 1 represented by their members $G(x)$, where all the quotients $V_{i}$ are elementary abelian 2-groups. 
Table 1 Exceptional $(n, 2)$-amalgams

\begin{tabular}{|c|c|c|c|c|c|c|c|}
\hline$n$ & $\mathcal{A}$ & $G(x) / G_{1}(x)$ & $V_{1}$ & $V_{2}$ & $V_{3}$ & $V_{4}$ & Some special completions \\
\hline \multirow[t]{5}{*}{3} & $\mathcal{A}_{3}^{(1)}$ & $L_{3}(2)$ & $2^{3}$ & & & & - \\
\hline & $\mathcal{A}_{3}^{(2)}$ & $L_{3}(2)$ & $2^{3}$ & & & & $M_{22}$ \\
\hline & $\mathcal{A}_{3}^{(3)}$ & $L_{3}(2)$ & $2^{3}$ & & & & - \\
\hline & $\mathcal{A}_{3}^{(4)}$ & $L_{3}(2)$ & $2^{3}$ & 2 & & & $\left(S_{8}: 2\right)^{+}$ \\
\hline & $\mathcal{A}_{3}^{(5)}$ & $L_{3}(2)$ & $2^{3}$ & 2 & & & $\operatorname{Aut}\left(M_{22}\right)$ \\
\hline \multirow[t]{5}{*}{4} & $\mathcal{A}_{4}^{(1)}$ & $L_{4}(2)$ & & & & & $M_{23}$ \\
\hline & $\mathcal{A}_{4}^{(2)}$ & $L_{4}(2)$ & $2^{6}$ & & & & $A_{64}$ \\
\hline & $\mathcal{A}_{4}^{(3)}$ & $L_{4}(2)$ & $2^{6}$ & $2^{4}$ & 2 & & $\mathrm{Co}_{2}$ \\
\hline & $\mathcal{A}_{4}^{(4)}$ & $L_{4}(2)$ & $2^{6}$ & $2^{4}$ & $2^{4}$ & & $J_{4}$ \\
\hline & $\mathcal{A}_{4}^{(5)}$ & $L_{4}(2)$ & $2^{6}$ & $2^{4}$ & $2^{4}$ & & $A_{256}$ \\
\hline \multirow[t]{2}{*}{5} & $\mathcal{A}_{5}^{(1)}$ & $L_{5}(2)$ & $2^{10}$ & & & & $J_{4}$ \\
\hline & $\mathcal{A}_{5}^{(2)}$ & $L_{5}(2)$ & $2^{10}$ & $2^{10}$ & $2^{5}$ & $2^{5}$ & $B M$ \\
\hline
\end{tabular}

\section{Some $(n, 3)$-examples}

The motivating examples for our project are the collinearity graphs of flag-transitive Petersen and Tilde geometries classified by Spectorov and the author in Ivanov (1998) and Ivanov and Shpectorov (2002). These and other known examples are combined in Table 2. In this table for a group $G$ acting locally projectively on a graph $\Gamma$ of type $(n, 3)$ we denote by $G_{\frac{1}{2}}(x)$ the largest subgroup in $G(x)$ which stabilizes every line passing through $x$ and put $V_{0}=G_{\frac{1}{2}}(x) / G_{1}(x)$. For $k \geq 1$, as in Table 1 , we put $V_{k}=G_{k}(x) / G_{k+1}(x)$ which is still elementary abelian except $V_{1}$ in Example 12 .

The entries $G$ for 11 a and 11 b contain the largest locally projective automorphism group of the Hamming graph $H(n, 3)$ and of the binary code, respectively. Some smaller groups also act locally projectively and they are easy to classify.

\section{Densely embedded and geometric subgraphs}

When dealing with locally projective amalgams we usually assume that the graph $\Gamma$ is a bipartite half of the coset graph of the universal completion of the corresponding amalgam. By the universality property, the coset graph is a tree with vertices in one half having valency $\left(2^{n}-1\right)$ and the vertices in the other half (the lines) having valency 3. The problem we consider is a pushing-up type problem for $L_{n}(2)$ and it is widely open with only a few special cases settled (cf. Parker and Rowley (1996)).

We define a densely embedded subgraph in the locally projective graph of type $(n, 3)$. 


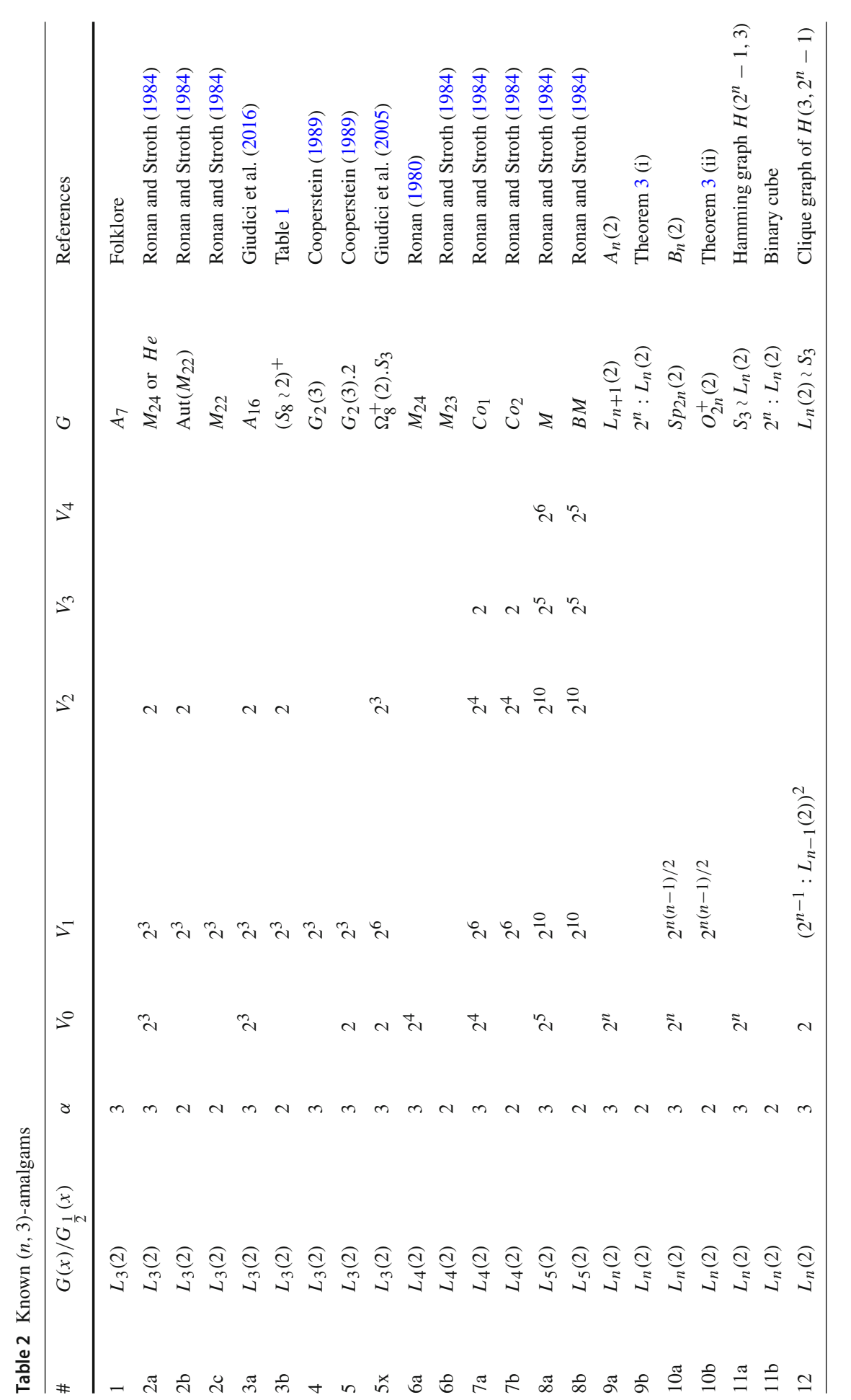


Definition 4 Suppose that $G$ acts locally projectively on $\Gamma$ of type $(n, 3)$ for $n \geq 3$, and let $\Delta$ be a connected subgraph in $\Gamma$. Then $\Delta$ is said to be densely embedded in $\Gamma$ if the following conditions hold:

(i) the subgroup $H$ of $G$ which stabilizes $\Delta$ as a whole induces on it a locally projective action of type $(n, 2)$, possibly with a non-trivial kernel;

(ii) if $x \in \Delta$ then $H(x)$ contains $G_{1}(x)$ and $H(x) / G_{1}(x)$ is an $L_{n}(2)$-complement to $G_{\frac{1}{2}}(x) / G_{1}(x)$ in $G(x) / G_{1}(x)$.

It is implicit in Definition 4 (ii) that a densely embedded subgraph exists only if $G(x) / G_{1}(x)$ splits over $G_{\frac{1}{2}}(x) / G_{1}(x)$. In fact densely embedded subgraphs exist quite often: in Table 2 the subgraph number $\mathrm{Nb}$ is densely embedded into the subgraph $\mathrm{Na}$ for

$$
N=2,3,6,7,8,9,10,11 \text {. }
$$

This observation served as a starting point for our classification project, which is still in progress. More specifically, we observed that, since both the $\mathrm{M}_{24}$ - and the $\mathrm{He}$ examples correspond to the same locally projective amalgam $2 \mathrm{a}$, and $M_{24}$ contains a densely embedded subgraph (stabilized by $\operatorname{Aut}\left(M_{22}\right)$ ), the universal cover of the Held graph must contain a densely embedded subgraph. In fact when the universal cover is folded onto the Held graph, the densely embedded subgraph folds onto the whole of the Held graph, so it is hard to see its traces in the $H e$-graph.

We turn the existence of densely embedded subgraphs into Theorem 16. Notice that in Table 2 there is only one instance when the action of $H$ on the corresponding densely embedded subgraph $\Delta$ is unfaithful (with kernel of order 2): $G$ is the Monster $8 \mathrm{a}$ and $H$ is the double cover of the Baby Monster $8 \mathrm{~b}$.

Next we introduce geometric subgraphs. For the classes of Petersen, Tilde and classical geometries the geometric subgraphs in the collinearity graphs enable to reconstruct the elements of higher types. Recall that every vertex $x$ is equipped with a $G F(2)$-vector space $\pi_{x}$, whose points are the lines containing $x$.

Definition 5 A connected subgraph $\Xi^{(k)}$ in $\Gamma$ is said to be geometric at level $k$, where $1 \leq k \leq n-1$, whenever together with an edge it always contains the line on this edge, and the following conditions hold:

(i) if $x \in \Xi^{(k)}$, then the set of neighbours $\Xi^{(k)}(x)$ of $x$ in $\Xi^{(k)}$ is a $k$-dimensional subspace in $\pi_{x}$ and the set-wise stabilizer of $\Xi^{(k)}(x)$ in $G(x)$ stabilizes $\Xi^{(k)}$;

(ii) the stabilizer $X^{(k)}$ of $\Xi^{(k)}$ in $G$ acts on $\Xi^{(k)}$ locally projectively with type $(k, 3)$ with kernel denoted by $K^{(k)}$.

It is clear that the geometric subgraphs at level 1 are just the lines, while the geometric subgraphs $\Xi^{(2)}$ at level 2 (called planes) are of valency 6 and the stabilizer $X^{(2)}$ of such a subgraph (containing $x$ and $l$ ) modulo its vertex-wise stabilizer $K^{(2)}$ is a completion of a Goldschmidt amalgam

$$
\mathcal{X}^{(2)}=\left\{X^{(2)}(x) / K^{(2)}, X^{(2)}(l) / K^{(2)}\right\},
$$


which is a locally projective amalgam of type $(2,3)$.

Usually a locally projective graph $\Gamma$ contains at least one family of planes (Example 4 contins three such families) and the simply connected version of $\Gamma$ contains a complete set of geometric subgraphs for all levels $k$ for $2 \leq k \leq n-1$. Since in the present work we are going to assume that $\Gamma$ contains planes anyway, we will not discuss the existence issue further. Instead we present a classical example of densely embedded and geometric subgraphs.

Example. Let $\left(V_{2 n}(2), q, f\right)$ be a $2 n$-dimensional $G F(2)$-space equipped with a nonsingular quadratic form $q$ of maximal Witt index $n$ whose associate bilinear form is $f$. Let $\Gamma=D P(n, 2)$ be the dual polar graph whose vertices are the maximal totally singular subspaces in $V_{2 n}(2)$ with respect to $f$, that is the $n$-dimensional subspaces $W_{n}$ in $V_{2 n}(2)$ such that $f(u, v)=0$ for any $u, v \in W_{n}$. Two vertices-subspaces are adjacent if their intersection has codimension 1 in each. This graph is locally projective of type $(n, 3)$ with respect to the symplectic group $G=S p_{2 n}$ (2) preserving $f$ (this is the graph 10a in Table 2). The subgraph $\Delta$ formed by the $n$-dimensional subspaces totally isotropic with respect to $q$ (the $n$-subspaces $U_{n}$ such that $q(u)=0$ for every $u \in U_{n}$ ) is the orthogonal graph (10b in Table 2) which is locally projective of type $(n, 2)$ with respect to the orthogonal group $H=O_{n}^{+}(2)$ and it is densely embedded in $D P(n, 2)$ with

$$
G(x) \cong 2^{n(n-1) / 2+n}: L_{n}(2) \text { and } H(x) \cong 2^{n(n-1) / 2}: L_{n}(2) .
$$

If $W_{n-k}$ is a totally singular subspace in $V_{2 n}(2)$ of dimension $n-k$, then the subgraph in $\Gamma$ formed by the vertices-subspaces containing $W_{n-k}$ is geometric at level $k$ isomorphic to $D P(k, 2)$. The planes $D P(2,2)$ are generalized quadrangles of order $(2,2)$ associated with $S p_{4}(2) \cong S_{6}$, which is a completion of the Goldschmidt amalgam $G_{3}^{1}=\left\{S_{4} \times 2, S_{4} \times 2\right\}$.

\section{Constructing densely embedded subgraphs}

In this section we establish the existence of densely embedded subgraphs under the following hypothesis.

Hypothesis $6(\Gamma, G)$ is a locally projective pair of type $(n, 3)$ for $n \geq 3$ satisfying the following conditions where $x$ is a vertex and $l$ is a line:

(H1) $\Gamma$ is simply connected so that the vertex-line graph $\Lambda$ is a tree, equivalently $G$ is the universal completion of the locally projective amalgam $\{G(x), G(l)\}$;

(H2) there is a G-orbit of planes realizing the Goldschmidt amalgam $G_{3}^{1}=\left\{S_{4} \times\right.$ $\left.2, S_{4} \times 2\right\}$

(H3) $G_{\frac{1}{2}}(x) / G_{1}(x)$ is the dual natural module $2^{n}$ of $G(x) / G_{\frac{1}{2}}(x) \cong L_{n}(2)$ and the extension splits:

$$
G(x) / G_{1}(x) \cong 2^{n}: L_{n}(2)
$$

(H4) $G_{1}(x) \neq 1$; 
(H5) $n \neq 4$.

Among the examples in Table 2, the following ones satisfy Hypothesis 6:

$$
2 a, 3 a, 7 a, 8 a, 10 a \text {. }
$$

They include the dual polar space graphs of $S p_{2 m}(2)$, the example completing in $A_{16}$ and the famous Mathieu-Conway-Monster sequence of tilde geometries. This again demonstrates the role of the Mathieu groups, as a path towards larger sporadic simple groups. Each of the above mentioned examples contains a densely embedded subgraph:

$2 b, 3 b, 7 b, 8 b, 10 b$,

respectively. In Example 6a all the conditions except (H4) and (H5) hold and the graph still contains a densely embedded subgraph $6 \mathrm{~b}$.

\section{1 $G_{1}(x)$ is a 2-group}

We start by stating the fundamental Thompson-Wielandt-Weiss Theorem (Weiss 1979) in its refined version in van Bon (2003) which we apply to the vertex-line graph $\Lambda$ of $\Gamma$ acted on by $G$.

Theorem 7 Let $\Lambda$ be a connected, finite, undirected graph, $\{x, l\}$ be an edge of $\Lambda$ and $G$ be a subgroup of Aut $(\Lambda)$ such that $G(v)^{\Lambda_{1}(v)}$ is primitive for $v=x$ and $l$. Let $\mu$ be the set of primes dividing the order of $G_{1}(x, l):=G_{1}(x) \cap G_{1}(l)$. Then either $|\mu|=1$ or there exists $p \in \mu$ such that either for $u=x$ and $v=l$ or for $u=l$ and $v=x$, we have $G_{1}(x, l)=G_{2}(u), G_{2}(v)$ is a $p$-group and $G_{2}(v)=G_{3}(v)$.

Next we present a lemma kindly offered by a referee of the present article.

Lemma 8 Assume that $\Gamma$ and $G$ satisfy the conditions $(i)-(v)$ in Definition 1 with $\alpha=3$. Let $x$ be a vertex of $\Gamma$. Then either $G_{1}(x)$ is a 2-group or $\left|G_{\frac{1}{2}}(x): G_{1}(x)\right| \leq 2$.

Proof. As above let $\Lambda$ be the vertex-line graph of $\Gamma$ and let $F$ denote edge-transitive action of $G$ on $\Lambda$. Let $\{x, l\}$ be an edge of $\Lambda$. By Definition 1 (iii) and (iv) the groups $F(z)^{\Lambda_{1}(z)}$, with $z \in\{x, l\}$ are primitive permutation groups. By Theorem 7 (where $p$ is clearly 2 for the considered situation) $F_{1}(x, l)$ is a 2-group or $F_{1}(x, l)=F_{2}(x)$ and $F_{2}(l)=F_{3}(l)$ is a 2-group or $F_{1}(x, l)=F_{2}(l)$ and $F_{2}(x)=F_{3}(x)$ is a 2-group. Hence $F_{2}(u)$ is a 2-group for at least one vertex $u \in\{x, l\}$. We can assume that $u=l$, since otherwise there will be nothing to prove. Assume now that for a vertex $x$ of $\Gamma$ the subgroup $F_{2}(x)$ is not a 2-group. Then $F_{1}(x, l)=F_{2}(x)$, and $\left|\Lambda_{1}(l)\right|=3$ gives $\left|F_{1}(x): F_{2}(x)\right|=\left|F_{1}(x): F_{1}(x, l)\right| \leq 2$.

It follows that if $x$ is a vertex of $\Gamma$, then $F_{2}(x)$ is a 2-group or $\left|F_{1}(x): F_{2}(x)\right| \leq 2$. Thus in the action of $G$ on $\Gamma, G_{1}(x)$ is a 2-group or $\left|G_{\frac{1}{2}}(x): G_{1}(x)\right| \leq 2$.

Example 12 in Table 2 is an example that $\left|G_{\frac{1}{2}}(x): G_{1}(x)\right|=2$ can occur with $G_{1}(x)$ not a 2-group. Hypothesis 6 (H3) excludes the possibility of $\left|G_{\frac{1}{2}}(x): G_{1}(x)\right| \leq$ 2 so we have the following. 
Corollary 9 Under Hypothesis $6 G_{1}(x)$ is a 2-group.

\subsection{On the stabilizer $G(I)$ of a line}

We keep assuming Hypothesis 6. For a line $l=\{x, y, z\}$ in $\Gamma$ put

$$
\Gamma(l)=\Gamma(x) \cup \Gamma(y) \cup \Gamma(z)
$$

(the set of vertices with minimal distance at most 1 from the vertices in $l$ ), let $G(l)$ be the set-wise stabilizer of $l$ in $G, G(x, y, z)$ be the vertex-wise stabilizer and

$$
G_{1}(l)=G_{1}(x) \cap G_{1}(y) \cap G_{1}(z)
$$

be the kernel of the action of $G(l)$ on $\Gamma(l)$. In this subsection we describe $G(l) / G_{1}(l)$ up to isomorphism and $G(l)^{\Gamma(l)}$ up to similarity. We start by describing the action of $G(l) \cap G(x)$ on $\Gamma(x)$.

Lemma 10 The following assertions hold:

(i) $G(x, y, z)^{\Gamma(x)}=Q: K$, where $K \cong L_{n-1}(2)$ and $Q \cong 2^{2(n-1)}$;

(ii) $Q$ contains exactly three copies $T_{1}, T_{2}$ and $T_{3}$ of the dual natural $K$-module;

(iii) the orbit lengths of $T_{1}$ on the set of lines other than l containing $x$ are 1 and that of $T_{2}$ and $T_{3}$ are 2 ;

(iv) every orbit of $Q$ on $\Gamma(x) \backslash l$ has length 4 and together with $l \backslash\{x\}$ it comprises the set of neighbours of $x$ in a plane containing $x$;

(v) $(G(l) \cap G(x))^{\Gamma(x)}$ is an extension of $G(x, y, z)^{\Gamma(x)}$ by an involution $\tau_{x} \in$ $G_{\frac{1}{2}}(x) / G_{1}(x)$ which commutes with $K$, swaps $y$ and $z$ and conjugates $T_{2}$ onto $T_{3}$.

Proof. Let $L=G L_{n+1}(2), V_{n+1}(2)$ be the natural module of $L$ and $v$ be a nonzero vector in $V_{n+1}(2)$. Then by (H3), $G(x)^{\Gamma(x)}$ is similar to the action of $L(v)$ on $V_{n+1}(2) \backslash\{0, v\}$, with $G_{\frac{1}{2}}(x)$ acting as the group of transvections with centre $v$. Then $G(x, y, z)^{\Gamma(x)}$ is the action of $L(v, u, w)$ on $V_{n+1}(2) \backslash\{0, v\}$, where $V_{2}:=\{0, v, u, w\}$ is a 2-subspace in $V_{n+1}$ (2) and (i) follows with $K$ corresponding to the stabilizer in $L(v, u, w)$ of an $(n-1)$-subspace $W$ in $V_{n+1}(2)$ disjoint from $V_{2}$. Now (ii) to (iv) follow with $T_{1}, T_{2}$ and $T_{3}$ being the groups of transvections with centres $v, u$ and $w$, respectively, and with axes containing $V_{2}$. Finally $\tau_{x}$ acts as the transvection with centre $v$ and axis $\langle W, v\rangle$. It is tempting to identify $v, u$ and $w$ with $x, y$ and $z$ right now. The former identification goes through the isomorphism $V_{n+1}(2) /\{0, v\} \cong \pi_{x}$ but the latter two have a limitation, since the action $G(x)^{\Gamma(x)}$ commutes with the involution which flips vertices in every line through $x$, and this involution does not correspond to an element of $L$. We will achieve an identication later when getting deeper inside $\Gamma$.

Let $M \cong L_{n}(2)$ denote the Levi complement in $G(x)^{\Gamma(x)} \cong 2^{n}: L_{n}(2)$ to $G_{\frac{1}{2}}(x)^{\Gamma(x)} \cong 2^{n}$ which in terms of the proof of Lemma 10 is the stabilizer in $L(v)$ of a hyperplane disjoint from $v$. 
Lemma 11 The following assertions hold:

(i) if $n \neq 3$, then $M$ represents the only class of complements and if $n=3$, there is an additional class whose representatives act transitively on $\Gamma(x)$;

(ii) if $n \neq 4$, then the complement $K$ as in Lemma 10 represents the only class of complements to $Q$ in $G(x, y, z)^{\Gamma(x)}$ and if $n=4$, then there are eight classes of such complements.

Proof. The result follows from the well known fact (Bell 1978) that the first cohomology group of $L_{n}(2)$ on its natural module is trivial unless $n=3$, in which case it is 1-dimensional.

By (H2), the graph $\Gamma$ contains planes locally isomorphic to the generalized quadrangle of order $(2,2)$, a basic property of which is reminded in the next lemma.

Lemma 12 Let $\Xi$ be the point graph of the generalized quadrangle of order $(2,2)$ and let $S \cong S_{6} \cong S p_{4}(2)$ be the automorphism group of $\Xi$. Then for a vertex $x \in \Xi$ the order of $S_{1}(x)$ is 2 and $S_{1}(x)$ permutes the two lines through $y \in \Xi(x)$ not containing $x$.

Proof. The vertices of $\Xi$ are the transpositions in $S_{6}$ with two transpositions adjacent if their supports are disjoint. The only nontrivial element of $S_{1}(x)$ is the vertextransposition $x$ itself. If $x=(12), y=(34)$ then the two lines containing $y$ but not $x$ (they are $\{(34),(15),(26)\}$ and $\{(34),(16),(25)\})$ are permuted by $x$.

By a general principle, the assertions in Lemma 12 hold for any faithful completion of the Goldschmidt amalgam

$$
\{S(x), S\{x, y\}\} \cong\left\{S_{4} \times 2, S_{4} \times 2\right\} \cong G_{3}^{1},
$$

acting locally projectively on the coset graph of the completion, particularly for the universal completion.

Lemma 13 The action $G_{1}(x)^{\Gamma(y)}$ is an elementary abelian 2-group which is the dual natural module for

$$
L_{n-1}(2) \cong G(x, y, z) / O_{2}(G(x, y, z)),
$$

and $G_{1}(x, y)^{\Gamma(z)}=1$.

Proof. By (H4), Corollary 9 and the transitivity of $G(x)$ on $\Gamma(x)$ we conclude that $G_{1}(x)^{\Gamma(y)}$ is a non-trivial normal 2-subgroup in $G(x, y)^{\Gamma(y)}$. The structure of the latter action follows from Lemma 10 with subgroups decorated by the superscipt $(y)$. By Lemma $12, G_{1}(x)^{\Gamma(y)}$ has orbits of length 2 both on $\Gamma(y) \backslash l$ and on the lines other than $l$ passing through $y$. By Lemma 10 (iii), (iv) we conclude that

$$
G_{1}(x)^{\Gamma(y)}=T_{i}^{(y)} \text { for } i=2 \text { or } 3
$$

and the claim follows. 


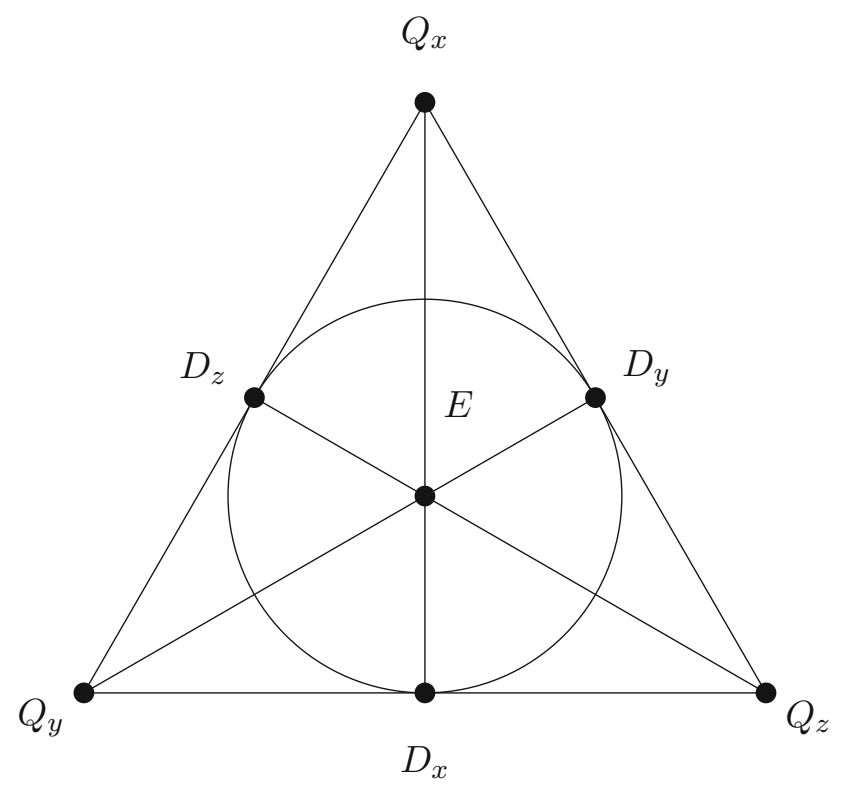

Fig. 1 Submodules

By Lemmas 10 and 13 we have the following (up to swapping of 2 and 3):

$$
T_{2}=G_{1}(y)^{\Gamma(x)}, \quad T_{3}=G_{1}(z)^{\Gamma(x)} .
$$

Now we can describe $G(x, y, x)^{\Gamma(l)}$ up to isomorphism.

Proposition 14 Under Hypothesis 6 the following assertions hold:

(i) $G(x, y, z)^{\Gamma(l)}=Q^{(l)}: K$, where $Q^{(l)}=O_{2}(G(x, y, z))^{\Gamma(l)}$ and $K \cong L_{n-1}(2)$;

(ii) $Q^{(l)}$ is elementary abelian of order $2^{3(n-1)}$ isomorphic to the direct product of $G_{1}(u)^{\Gamma(l)}$ 's taken for $u=x, y, z$;

(iii) each direct factor $G_{1}(u)^{\Gamma(l)}$ as in (ii) is the dual natural module of $K$ which acts faithfully on $\Gamma(v)$ for every $v \in l \backslash\{u\}$;

Proof. By Lemmas 10 and 13, the action of $Q^{(l)}$ on $\Gamma(u)$ is a direct product of dual natural modules of $G(x, y, z) / O_{2}(G(x, y, z)) \cong L_{n-1}(2)$ for every $u \in l$. Hence it only remains to justify that $G(x, y, z)$ splits over $Q^{(l)}$. But this is clear since for every $u \in l$ the action $G(x, y, z)^{\Gamma(u)}$ splits by Lemma 10 (i) and there is a symmetry between the vertices on $l$ performed by the setwise stabilizer $G(l)$.

The irreducible $K \cong L_{n-1}(2)$-submodules in $O_{2}\left(G(x, y, z)^{\Gamma(l)}\right)$ can be naturally labeled by the points of a Fano plane as on the Fig. 1 below, where $Q_{u}=G_{1}(u)^{\Gamma(l)}$ for $u \in l$ and the rest are various diagonal modules with $E$ acting as $G_{\frac{1}{2}}(u)$ on every $u \in l$. This picture enjoyed the obvious $S_{3}$-symmetry which is essential for the structure of $G(l)^{\Gamma(l)}$ to be revealed in our next result. 
Proposition 15 The group $G(l)^{\Gamma(l)}$ is a semidirect product of $G(x, y, z)^{\Gamma(l)}$ and an $S_{3}$-subgroup $R$ which permutes the vertices on $l$ and conjugates the direct factors $G_{1}(u)^{\Gamma(l)}$ for $u \in l$ in accordance. The subgroup $R$ is generated by a preimage in $G(l)$ of the involutions $\tau_{x}, \tau_{y}$ and $\tau_{z}$ as in Lemma $10(v)$ and which can be chosen to commute with a common $L_{n-1}(2)$-complement $K$ to $\mathrm{O}_{2}\left(G(x, y, z)^{\Gamma(l)}\right)$ in $G(x, y, z)^{\Gamma(l)}$.

Proof. Since $n \neq 4$ by (H5), by Lemma 11 all $L_{n-1}(2)$-complements to $Q^{(l)}$ in $G(x, y, z)^{\Gamma(l)}$ are conjugates of $K$. Then by considering the normalizer of $K$ in $G(l)^{\Gamma(l)}$ and applying a Frattini type argument we conclude that all the $\tau_{u}$ 's can be chosen to centralize $K$.

\subsection{The construction}

We are ready to prove our main result.

Theorem 16 Under Hypothesis $6 \Gamma$ contains a densely embedded subtree.

Proof. By (H3) we can find a subgroup $H(x)$ in $G(x)$ containing $G_{1}(x)$ such that $H(x) / G_{1}(x)$ is an $L_{n}(2)$-complement to $G_{\frac{1}{2}}(x) / G_{1}(x)$ in $G(x) / G_{1}(x) \cong 2^{n}: L_{n}(2)$. We always take $H(x)$ such that $H(x) / G_{1}(x)$ is the complement $M$ as in Lemma 11 so that $H(x)$ has wo orbits on $\Gamma(x)$. Then $H(x)$ is in fact the stabilizer in $G(x)$ of the ordered partition of $\Gamma(x)$ into the two $H(x)$-orbits. Thus $H(x) / G_{1}(x)$ is a Levi complement in terms of the proof of Lemma 10. Next we take $H(x, y, z):=H(x) \cap$ $G(x, y, z)$ which is the preimage of the maximal parabolic $P=2^{(n-1)}: L_{n-1}(2)$ in the action on $\Gamma(x)$. By Lemmas 10 and 13, we observe that $O_{2}(P)$ is $G_{1}(u)^{\Gamma(x)}$ for $u=y$ or $z$. The particular $u$ depends on the choice of the $L_{n}(2)$-complement $H(x) / G_{1}(x)$ and the conjugation of $H(x)$ by the preimage of an element from $G_{\frac{1}{2}}(x)$ which swaps $y$ and $z$, say by $\tau_{x}$, changes the vertex $u$ attached to $H(x)$. Therefore, without loss of generality we can assume that $u=y$. Then by Lemma 13, the preimage of $P$ in $G(x, y, z)$ (which is $H(x, y, z)$ ) is the direct product of $G_{1}(x) / G_{1}(l)$ and $G_{1}(y) / G_{1}(l)$ semidirecty multiplied by the complement $K$ which is normalized by the $S_{3}$-complement to $G(x, y, z) / G_{1}(l)$. Hence the normalizer of $H(x, y, z)$ in $G(l)$ picks up a preimage of the involution $\tau_{z}$.

Now we start defining $\Delta$ by putting into it $x$ and the $H(x)$-orbit on $\Gamma(x)$ which contains $y$. Then we define $H(y)$ as the conjugate of $H(x)$ by $\tau_{z}$ and the vertices of $\Gamma(y)$ to be put in $\Delta$ form the $H(y)$-orbit containing $x$. Then we proceed in the obvious way inductively on the distance from $x$. Suppose we know that a vertex $v$ is in $\Delta$, and also know the subgroup $H(v)$ which is a conjugate of $H(x)$ along the path joining $x$ with $v$. Then a vertex $w \in \Gamma(v)$ is contained in $\Delta$ if and only if $H(v, w)$ is not selfnormalized in $G\{v, w\}$. The result of this construction is uniquely determined by $H(x)$ and, therefore, is invariant under $H(x)$. The invariance under $N_{G(l)}(H(x, y, z))$ is also quite clear. The resulted graph $\Delta$ is a tree by (H1). The subgroup $H$ of $G$ generated by $H(x)$ and $\tau_{z}$ (equivalently by $H(x)$ and $N_{G(l)}(H(x, y, z))$ ) acts locally projectively on $\Delta$ with type $(n, 2)$. The group $H$ is the universal completion of the corresponding locally projective amalgam (cf. Neumann 1954). The geometric subgraphs intersected with $\Delta$ give geometric subgraphs in $\Delta$, which in turn force the action to be striclty 2 -arc transitive of collineation type. This completes the proof. 
Acknowledgements The author is indebted to anonymous referees for numerous suggestions including Lemma 8 and to Vladimir I. Trofimov for pointing out that the construction of densely embedded subgraphs should carry the uniqueness feature of the expansion as in the proof of Theorem 16. I would like to thank William Giuliano for most careful proofreading the paper.

Open Access This article is licensed under a Creative Commons Attribution 4.0 International License, which permits use, sharing, adaptation, distribution and reproduction in any medium or format, as long as you give appropriate credit to the original author(s) and the source, provide a link to the Creative Commons licence, and indicate if changes were made. The images or other third party material in this article are included in the article's Creative Commons licence, unless indicated otherwise in a credit line to the material. If material is not included in the article's Creative Commons licence and your intended use is not permitted by statutory regulation or exceeds the permitted use, you will need to obtain permission directly from the copyright holder. To view a copy of this licence, visit http://creativecommons.org/licenses/by/4.0/.

\section{References}

Bell, G.: On the cohomology of finite special linear groups I and II. J. Algebra 54, 216-238 and 239-259 (1978)

Cooperstein, B.: A finite flag-transitive geometry of extended $G_{2}$-type. Eur. J. Combin. 10, 313-318 (1989)

Djoković, D.Z., Miller, G.L.: Regular groups of automorphisms of cubic graphs. J. Combin. Theory (B) 29, 195-230 (1980)

Goldschmidt, D.M.: Automorphisms of trivalent graphs. Ann. Math. 111, 377-406 (1980)

Giudici, M., Li, C.H., Praeger, C.E.: Some locally 3-arc transitive graphs constructed from triality. J. Algebra 285, 11-28 (2005)

Giudici, M., Ivanov, A.A., Morgan, L., Praeger, C.E.: A characterisation of weakly locally projective amalgams related to $A_{16}$ and the sporadic simple groups $M_{24}$ and $\mathrm{He}$. J. Algebra 460, 340-365 (2016)

Ivanov, A.A.: Geometries of Sporadic Groups I. Petersen and Tilde Geometries. Cambridge University Press, Cambridge (1998)

Ivanov, A.A.: The Mathieu Groups. Cambridge University Press, Cambridge (2018)

Ivanov, A.A., Shpectorov, S.V.: Geometries of Sporadic Groups II: Representations and Amalgams. Cambridge University Press, Cambridge (2002)

Ivanov, A.A., Shpectorov, S.V.: Amalgams determined by locally projective actions. Nagoya Math. J. 176, 19-98 (2004)

Neumann, B.H.: An essay on free products of groups with amalgamations. Phios. Trans. R. Soc. Lond. Ser. A 246, 503-554 (1954)

Parker, C., Rowley, P.: On the non-commuting case for $\left(S_{3}, L_{3}(2)\right)$-amalgams. J. Algebra 181, 267-285 (1996)

Ronan, M.: Covering and automorphisms of chamber systems. Eur. J. Comb. 1, 259-269 (1980)

Ronan, M.A., Stroth, G.: Minimal parabolic geometries for the sporadic groups. Eur. J. Combin. 5, 59-91 (1984)

Sims, C.C.: Graphs and finite permutation groups. Math. Z. 95(1), 76-86 (1967)

Trofimov, V.I.: Vertex stabilizers of locally projective groups of automorphisms of graphs. A summary. In: Groups, Combinatorics and Geometry (Durham, 2001)

van Bon, J.: Thompson-Wielandt like theorems revisited. Bull. Lond. Math. Soc. 35, 30-36 (2003)

Weiss, R.: Elations of graphs. Acts Math. Scient. Hungary 34, 101-103 (1979)

Publisher's Note Springer Nature remains neutral with regard to jurisdictional claims in published maps and institutional affiliations. 